\title{
Real-World Treatment Patterns for Golimumab and Concomitant Medications in Japanese Rheumatoid Arthritis Patients
}

\author{
Masateru Okazaki (D) Hisanori Kobayashi · Yutaka Ishii • \\ Masayoshi Kanbori · Tsutomu Yajima
}

Received: December 25, 2017 / Published online: February 22, 2018

(C) The Author(s) 2018. This article is an open access publication

\begin{abstract}
Introduction: The aim of this study was to investigate real-world treatment patterns for use of golimumab and concomitant medications in Japanese patients with rheumatoid arthritis.

Methods: This study was a post hoc retrospective analysis from post-marketing surveillance data on 2350 Japanese patients with moderate/severe rheumatoid arthritis who received golimumab for 24 weeks. The study population was divided based on initiation treatment or dose adjustment patterns with golimumab, methotrexate, or oral glucocorticoids.

Results: Logistic regression analysis revealed that the baseline factors associated with administration of golimumab (100 mg) were
\end{abstract}

Enhanced content To view enhanced content for this article go to https://doi.org/10.6084/m9.figshare. 5831736.

M. Okazaki $(\varangle) \cdot$ H. Kobayashi · Y. Ishii Immunology Department, Medical Affairs Division, Janssen Pharmaceutical K.K., Tokyo, Japan e-mail: mokazak1@its.jnj.com

M. Kanbori

Japan Safety and Surveillance Division, Research and Development Division, Janssen Pharmaceutical K.K., Tokyo, Japan

\section{T. Yajima}

Biostatistics Department, Research and Development Division, Janssen Pharmaceutical K.K., Tokyo, Japan higher body weight, failure of prior biological therapy (bio-failure), no previous methotrexate use, and respiratory disease, while previous methotrexate use and absence of renal impairment or respiratory disease were associated with concomitant methotrexate therapy, and previous glucocorticoid use was associated with concomitant glucocorticoid therapy. The following associations were identified with regard to dose adjustment during treatment: bio-failure, no previous methotrexate use, previous csDMARDs use, presence of respiratory disease, allergy history, and higher CRP for golimumab dose escalation; shorter disease duration, previous GC, and no previous methotrexate use for methotrexate dose escalation; no prior biological therapy and renal impairment for methotrexate dose reduction; no previous GC use for glucocorticoid dose escalation; and absence of Steinbrocker's stage II/III/IV, absence of Steinbrocker's class II, no bio-failure, and no previous csDMARDs use for glucocorticoid dose reduction.

Conclusions: This study revealed that various baseline factors were associated with initiation of treatment and dose adjustment of golimumab, methotrexate, or oral glucocorticoids, reflecting both the treatment strategies of physicians for improving RA symptoms and/or reducing adverse events.

Funding: Janssen Pharmaceutical K.K. and Mitsubishi Tanabe Pharma Corporation. 
Keywords: Glucocorticoid; Methotrexate; Post-marketing Rheumatoid arthritis

\section{INTRODUCTION}

Rheumatoid arthritis (RA) is a chronic inflammatory autoimmune disease that causes progressive joint destruction and various morbidities, as well as increasing mortality. In Japan, the prevalence of RA is estimated to be $0.6-1.0 \%$ [1]. Biological disease-modifying antirheumatic drugs (bDMARDs) can be used to achieve low disease activity or even remission of moderate-to-severe RA [2, 3]. Golimumab (GLM) is a bDMARD that targets a unique epitope on the tumor necrosis factor- $\alpha$ (TNF- $\alpha$ ) molecule. It binds to both the soluble and membrane-bound forms of TNF- $\alpha$ with a high affinity, creating stable complexes that prevent the binding of TNF- $\alpha$ to its receptors $[4,5]$. Based on this mechanism of action, GLM is indicated for the treatment of patients with active RA [6], psoriatic arthritis [7], ankylosing spondylitis [8], and active nonradiographic axial spondyloarthritis [9], two clinical trials of GLM, GO-FORTH [10] and GO-MONO [11], have been conducted in Japanese patients with active RA. Based on the results, two GLM dose levels have been approved by the Pharmaceuticals and Medical Devices Agency (PMDA), which are $50 \mathrm{mg}$ of GLM combined with methotrexate (50 mg GLM + MTX) or $100 \mathrm{mg}$ of GLM either combined with methotrexate (100 mg GLM + MTX) or as monotherapy (100 mg GLM-mono). After GLM was marketed, a large post-marketing surveillance (PMS; UMIN000015895) was conducted to evaluate its safety and effectiveness for Japanese RA patients in real-world practice [12]. GLM showed a comparable safety and efficacy profile in the real-world setting to phase II/III clinical trials, but medications such as methotrexate and/or oral glucocorticoids were often used concomitantly with GLM in routine practice.

According to the recent treatment strategy for RA proposed by the European League Against Rheumatism (EULAR) [13], it is recommended that glucocorticoids (GC) should be gradually reduced and ultimately stopped, usually within 3 months from the start of treatment and only occasionally by 6 months because of many potential risks. On the other hand, while combined therapy with bDMARDs and methotrexate (MTX) achieves better results than monotherapy with biological agents [14-17], MTX has numerous adverse effects such as stomatitis, alopecia, skin rashes, leukopenia, thrombocytopenia, anemia, liver disorder, and gastrointestinal pulmonary, and central nervous system symptoms [18]. Accordingly, many RA patients and their physicians wish to taper or discontinue MTX therapy. However, the current treatment patterns for use of GLM and concomitant medications in Japanese RA patients are unclear, and there is no established evidence, so concomitant therapy is decided empirically by physicians depending on patient characteristics.

Against this background, we investigated how physicians selected regimens and modified the dosages of concomitant drugs when using GLM to treat moderate-to-severe RA in realworld practice in Japan. To do this, we performed a post hoc subgroup analysis of PMS data that assessed current practice and factors associated with treatment patterns in patients with active RA receiving GLM for 24 weeks with or without concomitant medications.

\section{METHODS}

\section{Data Source}

The present study was a post hoc retrospective analysis of PMS data (UMIN000015895) collected from Japanese patients with RA who received GLM therapy between September 2011 and May 2013. The PMS was an open-label, non-interventional, observational study conducted at selected medical facilities across Japan, in which patients were followed for 24 weeks. The study protocol and ethical considerations were assessed by the institutional review board of each study center and this study was also endorsed by the Japanese PMDA. This PMS was conducted under the Japanese regulations (Ministry of Health, Labor and Welfare 
Ministerial Ordinance no. 171) for Good PostMarketing Study Practice (GPSP), and was initiated after a contract with each study center. This article is based on previously conducted post-marketing surveillance study and does not contain any new interventional studies with human participants or animal subjects performed by any of the authors.

\section{Patients Enrolled in this Analysis}

This analysis included 2350 patients from the PMS efficacy dataset who underwent evaluation of RA disease activity using the 28-joint disease activity score [19] combined with C-reactive protein (DAS28CRP) at both baseline and final evaluations, who had moderate disease activity (MDA; DAS28CRP $>3.2$ and $\leq 5.1$ ) or high disease activity (HDA; DAS28CRP $>5.1$ ) at baseline, and who had no more than one dose change of GLM during the study period (Fig. 1).

\section{Factors Investigated}

The following information at baseline was retrieved from the PMS database: sex, age, body weight, smoking history, duration of RA, disease activity, Steinbrocker's radiographic stage and functional class [20], previous treatment (GC, MTX, and other csDMARDs), comorbidities (renal impairment, hepatic dysfunction, and respiratory disease), allergy history, DAS28CRP, CRP, patient's overall assessment on a visual analog scale (patient VAS), simple disease activity index (SDAI), and clinical disease activity index (CDAI). Respiratory disease includes asthma, atelectasis, cough, eosinophilic pneumonia, interstitial lung disease, pleurisy, pneumonia aspiration, productive cough, rhinorrhoea, upper respiratory tract inflammation, and oropharyngeal pain.

\section{Statistical Analysis}

Statistical analysis was performed using R software (version 3.2.5) [21] without imputation of missing data. Categorical variables were summarized as frequencies and proportions (\%), while continuous variables were summarized as the mean and standard deviation (SD) unless otherwise mentioned. In all analyses, a twosided $p$ value $<0.05$ was considered to indicate statistical significance. Paired two-group comparisons were performed by using the paired $t$ test for continuous variables. Student's $t$ test or Welch's $t$ test was employed to compare

\begin{tabular}{l}
\hline Total patients registered \\
\cline { 2 - 4 }
\end{tabular}

Fig. 1 Flowchart of this study 
unpaired continuous variables, and Fisher's exact test (Monte Carlo version) was used for comparison of unpaired categorical data. Continuous variables were compared between groups with ANOVA or the Kruskal-Wallis test, while the Tukey-Kramer test was used to compare multiple continuous variables within groups.

\section{Evaluation of Treatment Initiation for Use of GLM and Concomitant Medications}

The patterns for initiation of treatment with GLM and concomitant medications were analyzed by dividing the patients into subgroups (A: $50 \mathrm{mg}$ GLM vs. $100 \mathrm{mg}$ GLM, B: concomitant use of MTX vs. no MTX, and C: concomitant use of GC vs. no GC) and the demographic profile of each subgroup was evaluated. Then the patient characteristics were analyzed to identify baseline factors associated with each treatment pattern.

\section{Evaluation of Dose Adjustment and its Outcome}

The patterns of dose adjustment for GLM and concomitant drugs during the study period were also investigated by dividing the patients into subgroups based on changes in the use of GLM, MTX, or GC (A continuing $50 \mathrm{mg}$ of GLM vs. dose escalation of GLM; B continuing $100 \mathrm{mg}$ of GLM vs. dose reduction of GLM; C continuing the same dose of MTX vs. dose escalation or dose reduction of MTX; and D continuing the same dose of GC vs. dose escalation or dose reduction of GC). In addition, patient characteristics were analyzed to identify baseline factors associated with each dose adjustment pattern. The clinical response to dose adjustment was also evaluated by using changes of the DAS28CRP score ( $\triangle \mathrm{DAS} 28 \mathrm{CRP})$ and was compared among the subgroups.

\section{Association of Baseline Factors with Each Treatment Pattern}

Factors showing an association with the patterns of treatment initiation or dose adjustment were investigated by performing univariate and multivariate logistic regression analyses that included patient characteristic variables. Univariate logistic regression analysis was performed with patient demographic factors that showed $p<0.10$ in other analyses, and multivariate analysis was subsequently conducted by using variables that showed $p<0.20$ in the univariate analysis.

\section{RESULTS}

\section{Initiation of GLM and Concomitant Medications}

Table 1 shows the demographic data for each subgroup of patients depending on the pattern for initiation of treatment with GLM, MTX, or GC. The initial dose of GLM was $50 \mathrm{mg}$ in 1981 patients (84.3\%) and $100 \mathrm{mg}$ in 369 patients $(15.7 \%)$. There were baseline differences between these GLM dose subgroups (50 vs. $100 \mathrm{mg}$ ) with respect to age, body weight, disease activity, previous treatment [prior biological therapy (bio-experience), failure of prior biological therapy (bio-failure), MTX, and other csDMARDs], comorbidities (renal impairment, and respiratory disease), allergy history, DAS28CRP, CRP, SDAI, and CDAI. With regard to concomitant MTX treatment, 1852 patients (78.8\%) received MTX and 498 patients (21.2\%) did not. These subgroups (with MTX vs. without MTX) showed baseline differences of age, disease duration, disease activity, Steinbrocker's stage/class, previous treatment (bio-experience, bio-failure, GC, MTX, and other csDMARDs), comorbidities (renal impairment and respiratory disease), allergy history, DAS28CRP, CRP, SDAI, and CDAI. A total of 1219 patients (51.9\%) received concomitant GC treatment and 1131 patients $(48.1 \%)$ did not receive GC treatment. Baseline differences between the two GC subgroups (with GC vs. without GC) were noted with respect to Steinbrocker's stage/class, previous treatment (bio-experience, bio-failure, GC, MTX, and other csDMARDs), comorbidities (renal impairment, hepatic dysfunction, and respiratory disease), DAS28CRP, CRP, and SDAI. 


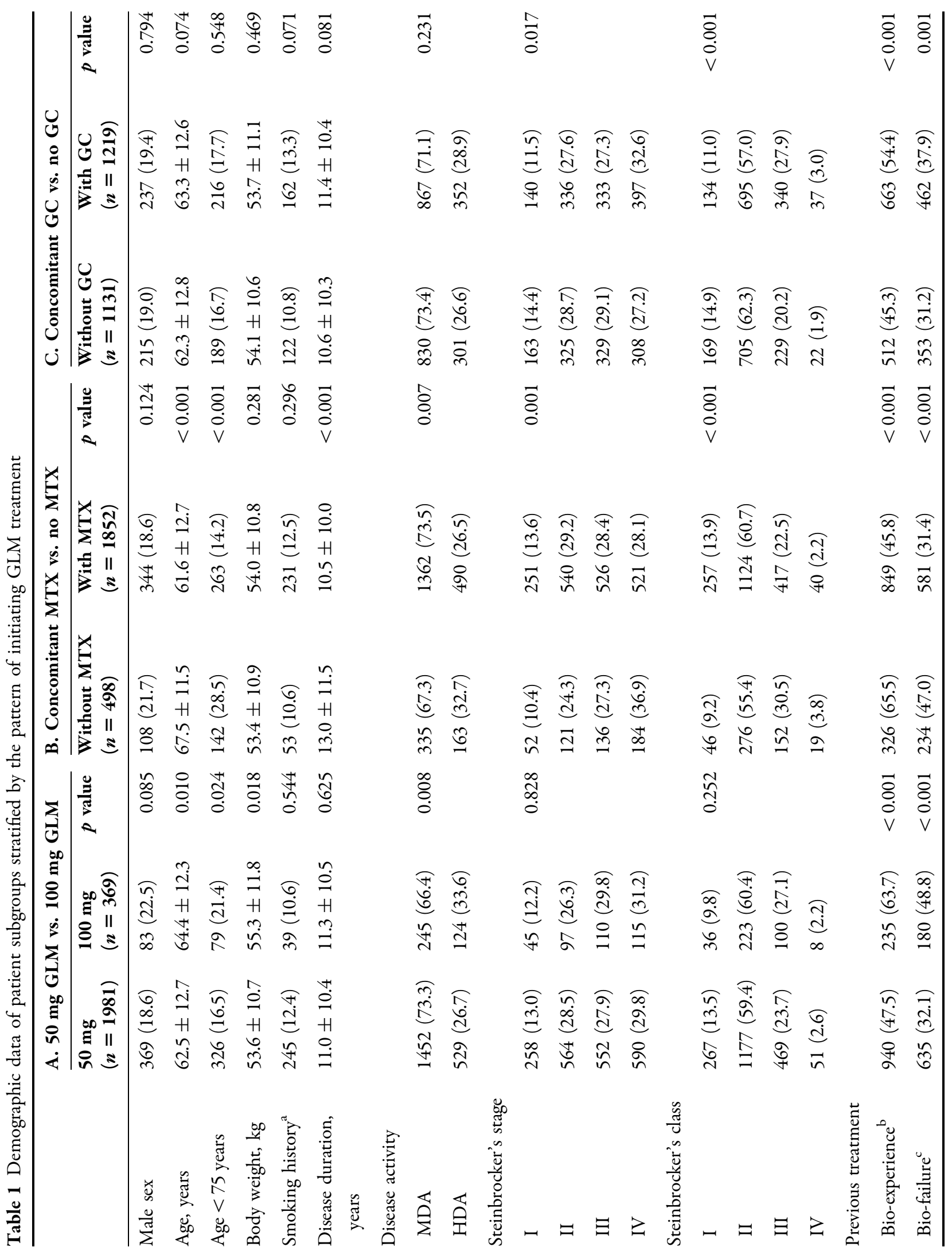




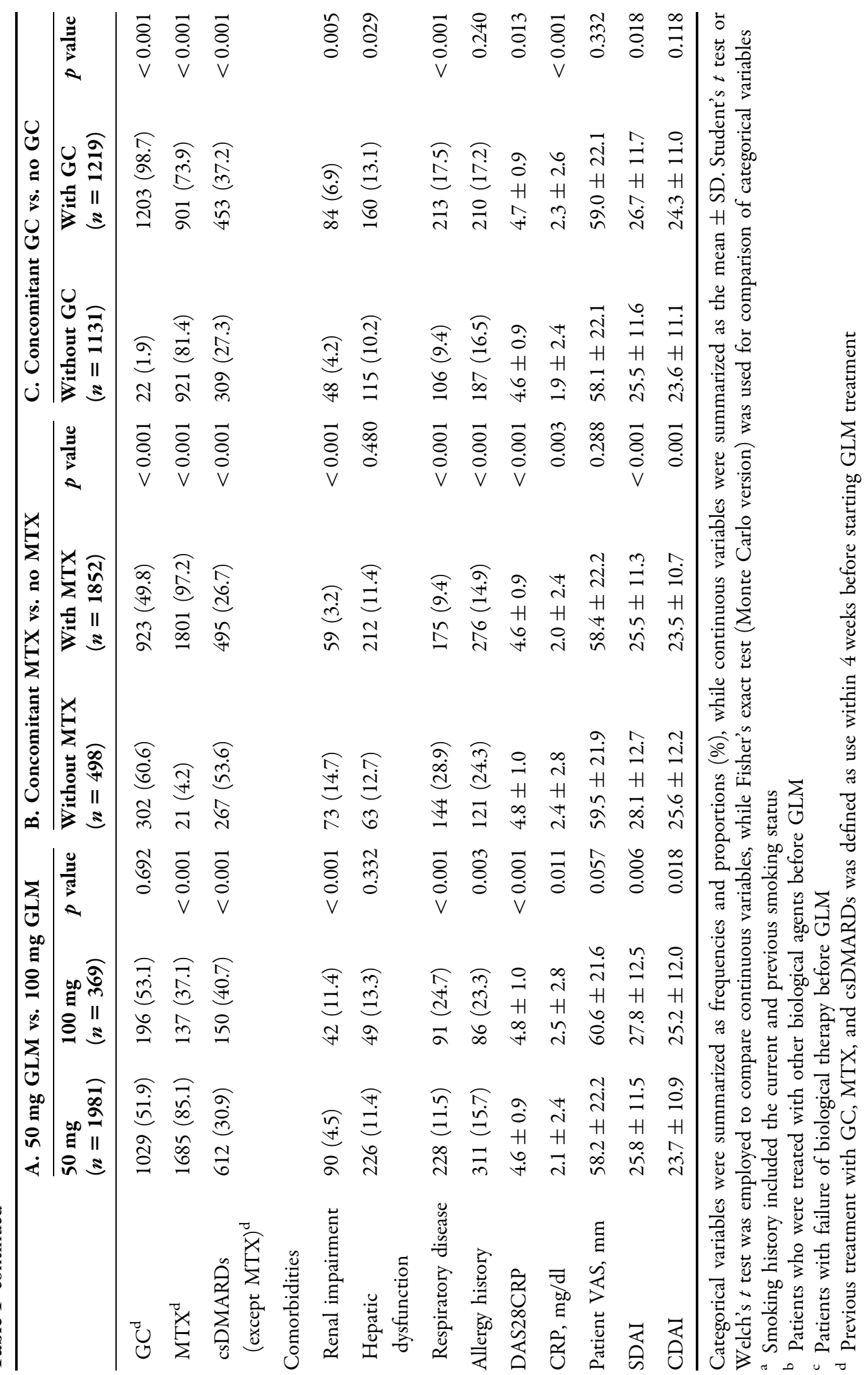


Multivariate logistic analysis was performed to identify factors associated with the initial GLM dose, concomitant MTX treatment, and concomitant GC treatment (Fig. 2). Factors associated with selecting $100 \mathrm{mg}$ of GLM as initial treatment [adjusted odds ratio (OR), 95\% confidence interval $(\mathrm{CI}), p<0.05$ ] were body weight $(1.019,1.007-1.032)$, bio-failure (1.448, 1.100-1.906), previous MTX use $(0.103$, $0.077-0.139)$, and respiratory disease (1.645, 1.181-2.293) (Fig. 2a). Factors associated with concomitant administration of MTX were previous MTX use (804.232, 461.587-1401.231), renal impairment $(0.251,0.098-0.643)$, and respiratory disease $(0.281,0.142-0.553)$ (Fig. 2b). Only one factor was associated with concomitant administration of GC, which was previous GC use (4352.473, 2164.187-8753.414) (Fig. 2c).

\section{Dose Adjustment Patterns and Outcome}

Dose adjustment patterns were evaluated for GLM, MTX, and GC during the study period. The dose of GLM was increased from 50 to $100 \mathrm{mg}$ in 327 patients, while it was reduced from 100 to $50 \mathrm{mg}$ in 21 patients (Table 2). Dose escalation of MTX was performed in 211 patients, while 178 patients received dose reduction. The average dose of MTX at initiation of treatment (the average for 4 weeks after starting GLM) was $8.13 \pm 3.09 \mathrm{mg} /$ week and it was slightly higher at $8.18 \pm 3.17 \mathrm{mg} /$ week by the end of the study period (the average for the last 4 weeks of the study), but the difference was not significant $(p=0.064)$. Dose escalation of GC was performed in 77 patients and dose reduction was done in 373 patients. The average GC dose was $4.81 \pm 2.75 \mathrm{mg} /$ day at initiation of treatment and it was significantly decreased to $4.49 \pm 2.62 \mathrm{mg} /$ day at the end of the study period $(p<0.001)$.

Next, the patient background factors associated with the dose adjustment patterns for GLM, MTX, and GC were assessed by univariate and multivariate regression analyses to identify significant differences among the subgroups of patients. Various factors were associated with dose escalation of GLM from 50 to $100 \mathrm{mg}$, which were bio-failure $(2.499,1.933-3.230)$, previous MTX use $(0.686,0.494-0.952)$, previous other csDMARDs use $(1.364,1.042-1.787)$, respiratory disease $(1.451,1.017-2.070)$, allergy history (1.421, 1.031-1.958), and CRP (1.073, 1.024-1.124) (Fig. 3a). On the other hand, no factors were associated with dose reduction of GLM from 100 to $50 \mathrm{mg}$. Patient background factors associated with dose escalation of MTX were disease duration (0.971, 0.947-0.997), previous GC use (1.451, 1.008-2.087), and previous MTX use (0.121, 0.063-0.232) (Fig. 3b), while the factors associated with dose reduction of MTX were bio-experienced (0.517, $0.343-0.779$ ) and renal impairment (3.312, 1.461-7.508). Although only previous GC use was associated with dose escalation of GC (0.031, 0.011-0.094) (Fig. 3c), various background factors were associated with dose reduction of GC, including Steinbrocker's stage II $(0.510,0.320-0.813)$, Steinbrocker's stage III $(0.532,0.327-0.867)$, Steinbrocker's stage IV $(0.529,0.323-0.869)$, Steinbrocker's class II (0.588, 0.371-0.931) bio-failure (0.446, $0.296-0.674)$, and previous csDMARDs use $(0.747,0.558-0.999)$.

\section{Clinical Response and Dose Adjustment Pattern}

The clinical response was compared among subgroups of patients based on the dose adjustment patterns for GLM, MTX, and GC during the study period. With regard to dose adjustment of GLM, $\triangle$ DAS28CRPs showed a significant difference between continuing $50 \mathrm{mg}$ of GLM vs. continuing $100 \mathrm{mg}$ of GLM, between continuing $50 \mathrm{mg}$ of GLM vs. dose escalation of GLM, and between continuing $100 \mathrm{mg}$ of GLM vs. dose escalation of GLM (Fig. 4a). In relation to MTX, $\triangle$ DAS28CRPs showed a significant difference between dose escalation of MTX vs. dose reduction of MTX (Fig. 4b). Regarding GC treatment, $\triangle$ DAS28CRPs showed a significant difference between continuing the same dose of GC vs. dose reduction of GC and between dose escalation of GC vs. dose reduction of GC (Fig. 4c). 
(a) Selection of $100 \mathrm{mg}$ GLM

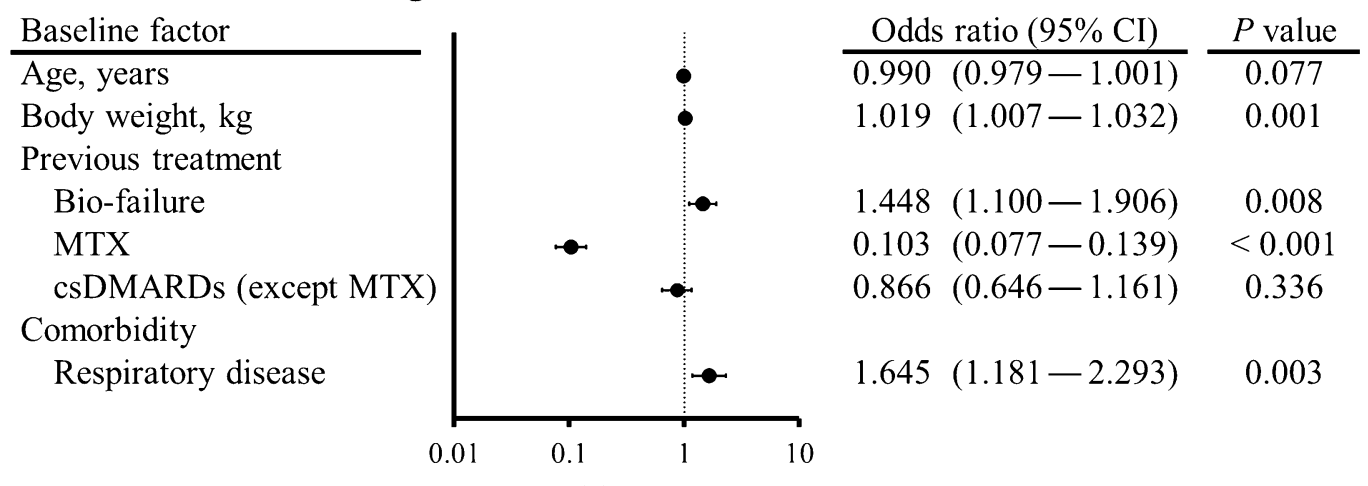

\section{(b) Selection of MTX}

\begin{tabular}{l} 
Baseline factor \\
\hline Age, years \\
Steinbrocker's stage \\
I \\
II \\
III \\
IV \\
Previous treatment \\
MTX \\
Comorbidity \\
Renal impairment \\
Respiratory disease
\end{tabular}

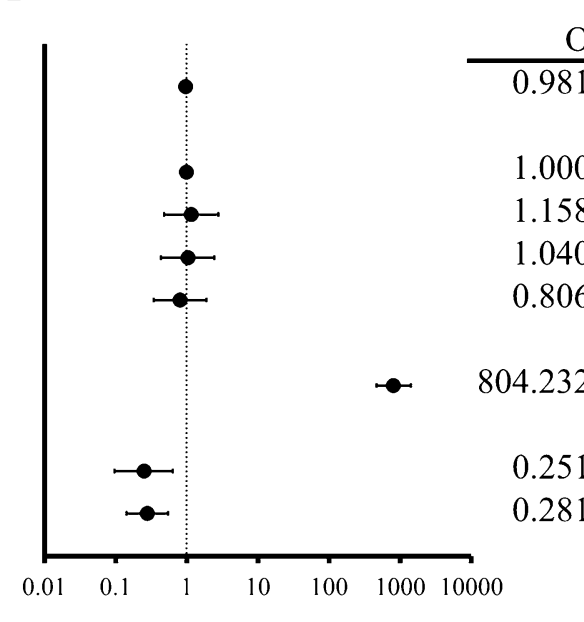

$\frac{\text { Odds ratio }(95 \% \mathrm{CI})}{0.981} \frac{P \text { value }}{0.064}$

$1.000 \quad$ (reference)

$1.158 \quad(0.483-2.776) \quad 0.742$

$1.040 \quad(0.437-2.477) \quad 0.929$

$0.806 \quad(0.343-1.891) \quad 0.620$

Previous treatment

Renal impairment

Respiratory disease

\section{(c) Selection of GC}

\section{Odds ratio}

\begin{tabular}{l} 
Baseline factor \\
\hline Age, years \\
Previous treatment \\
GC \\
Comorbidity \\
Respiratory disease \\
DAS28CRP \\
CRP, mg/dL
\end{tabular}

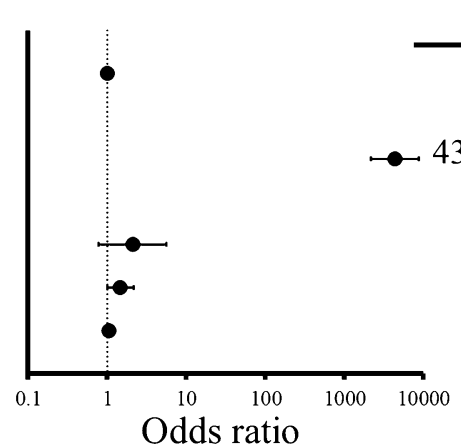

$\frac{\text { Odds ratio }(95 \% \mathrm{CI})}{1.005(0.979-1.031)} \frac{P \text { value }}{0.732}$

$4352.473(2164.187-8753.414)<0.001$

2.099

$(0.780-5.653)$

0.142

1.466

$(0.999-2.152)$

0.051

1.041

$(0.905-1.198)$

0.572

Odds ratio

Fig. 2 Forest plots illustrating patient background factors associated with selection of $100 \mathrm{mg}$ of golimumab (a), concomitant use of methotrexate (b), and glucocorticoids (c) at the initiation of golimumab treatment. Univariate logistic regression analysis was performed using demographic factors that showed $p<0.10$ in other analyses, after which multivariate analysis was conducted by using variables showing $p<0.20$ in the univariate analysis. Error bars indicate the $95 \%$ confidence interval (CI) 


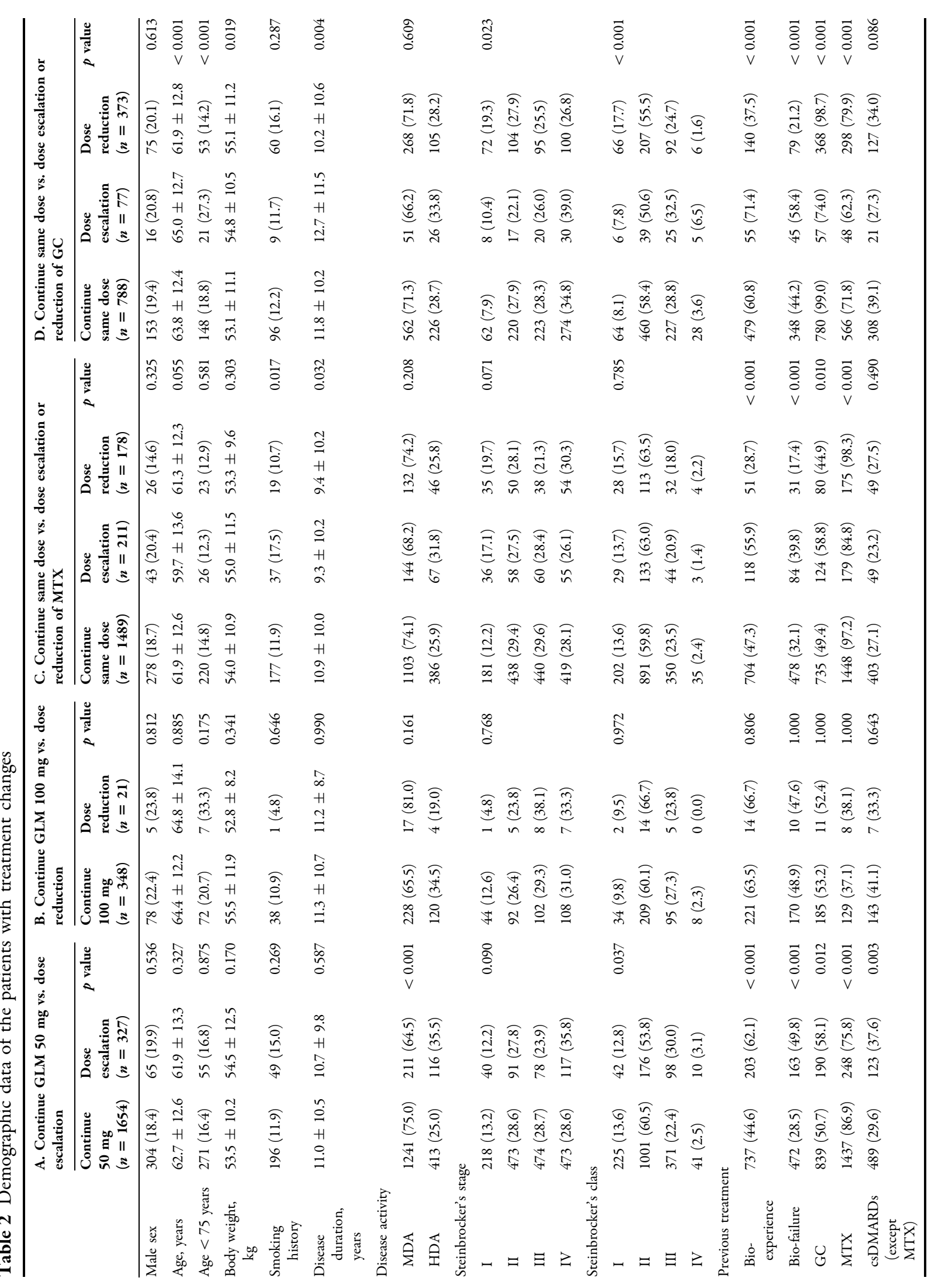




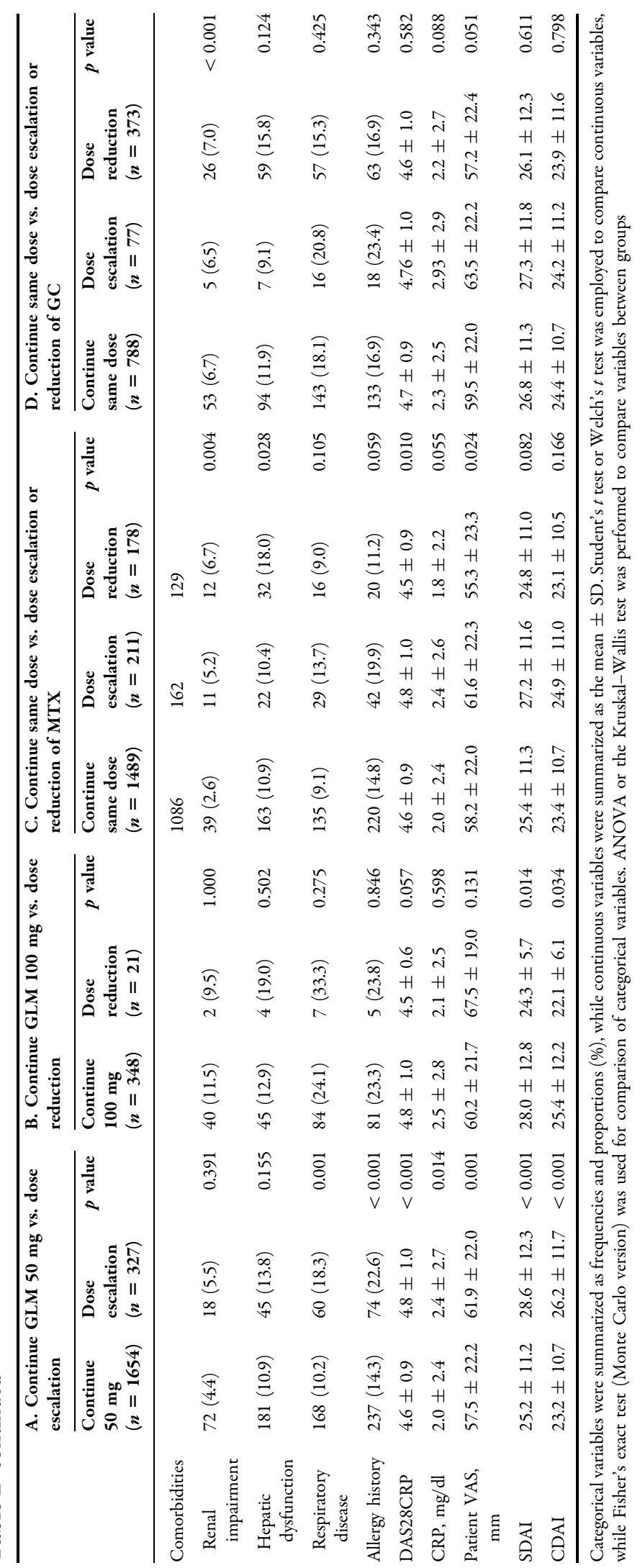




\section{DISCUSSION}

This study investigated the real-world treatment patterns for GLM and concomitant medications in Japanese RA patients. In addition, the factors associated with each treatment pattern were determined. Initiation of treatment with $100 \mathrm{mg}$ of GLM was associated with no previous use of MTX and with the presence of respiratory disease, which were also factors that influenced the selection of concomitant MTX therapy. Because GLM monotherapy is only approved at a dose of $100 \mathrm{mg}$ in Japan, it could be expected that MTX-naïve patients would be selected to receive this GLM dose as monotherapy. In fact, almost half of the MTX-naive patients received $100 \mathrm{mg}$ of GLM as monotherapy (43.4\%, 229/528). Body weight and bio-failure were also associated with selecting $100 \mathrm{mg}$ of GLM. Body weight is known to affect the pharmacokinetics of monoclonal antibodies after subcutaneous injection, and weight has been reported to influence the apparent clearance and volume of distribution of GLM $[22,23]$. Also, it was previously reported that GLM $100 \mathrm{mg}+$ MTX prevents joint damage more effectively in Japanese patients with active radiographic progression than GLM $50 \mathrm{mg}+$ MTX [10]. Therefore, physicians may have been more likely to select $100 \mathrm{mg}$ of GLM for patients with a higher body weight and bio-failure.

Dose escalation of GLM was performed in 327 patients, and was associated with the baseline factors of bio-failure, previous non-use of MTX, previous use of other csDMARDs, respiratory disease, allergy history, and higher CRP. The dose escalation rate was higher in the subgroup starting treatment with $50 \mathrm{mg}$ of GLM as monotherapy $(30.7 \%, 79 / 257)$ than in the subgroup receiving $50 \mathrm{mg}$ of GLM + MTX (14.4\%, 248/1724). This finding suggested that dose escalation was performed to improve the control of RA symptoms in patients with higher CRP, previous bio-failure and/or patients receiving a low dose of GLM as monotherapy. In fact, the clinical response was worse in the patients receiving dose escalation of GLM to $100 \mathrm{mg}$ than in the subgroup remaining on $50 \mathrm{mg}$. Because GLM monotherapy at a dose of
$50 \mathrm{mg}$ is not approved in Japan, dose escalation might also have been performed to reduce medical costs (Japanese national health insurance only subsidizes approved indications). On the other hand, the rate of dose reduction of GLM was lower $(5.7 \%, 21 / 369)$ than dose escalation of GLM $(16.5 \%, 327 / 1981)$. It might be the result that the use of $100 \mathrm{mg}$ GLM did not cause any problem like safety and/or medical cost.

Previous use of MTX was associated with continuation of MTX at initiation of GLM treatment, and almost all of the patients who were already using MTX were placed on MTX + GLM (98.9\%, 1801/1822). It was also found that the average dose of MTX at initial treatment was low compared to other countries [24], because the dose upper limitation of MTX was $8 \mathrm{mg} /$ week by $2011 \mathrm{in} \mathrm{Japan} \mathrm{[25]} \mathrm{and} \mathrm{it} \mathrm{was}$ recommended to start $8 \mathrm{mg} /$ week by a guideline of MTX use for the RA patients [26]. These results suggested that Japanese physicians employed GLM as add-on therapy for MTX at low dose according to the EULAR recommendation and Japan guidelines [13, 26].

Absence of renal impairment and absence of respiratory disease were also associated with concomitant use of MTX + GLM. This was probably because MTX is known to cause nephrotoxicity [27] and lung disease [28]. In addition, bio-experience at baseline and renal impairment were associated with MTX dose reduction, while shorter disease duration, previous GC use, and no previous MTX use were associated with MTX dose escalation. $\triangle$ DAS28CRP was higher in the subgroup with MTX dose reduction than in the patients with MTX dose escalation, suggesting that physicians might have reduced the dose of MTX when RA symptoms improved, especially in bio-naïve patients. On the other hand, the dose of MTX might be escalated if symptoms persisted, especially in the patients with shorter disease durations, previous GC, and/or no previous MTX use. This result also suggested that GC treatment might be selected as a first choice for early RA and sequentially MTX treatment might be added on. It followed EULAR recommendation for early RA treatment, in which short-term low dose of GCs and MTX with dose 


\section{(a) Dose escalation of GLM}

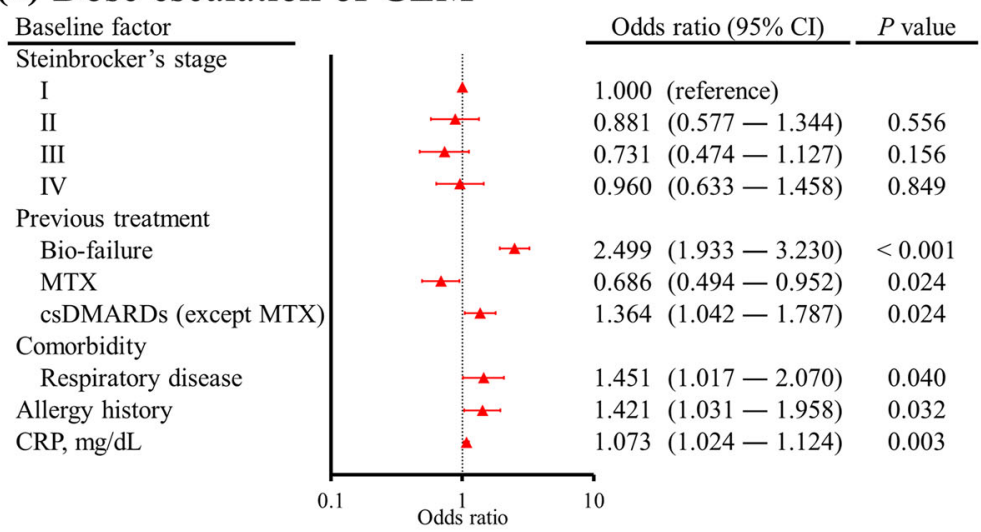

(b) Dose escalation/reduction of MTX

Baseline factor
Age, years
Smoking history
Disease duration, years
Steinbrocker's stage
I
II
III
IV
Brevious treatment
GC
MTX
Comorbidity
Renal impairment
Hepatic dysfunction
DAS28CRP
CRP, mg/dL
SDAI

(c) Dose escalation/reduction of GC

\begin{tabular}{ll}
\multicolumn{2}{c}{ Dose escalation of MTX } \\
\cline { 1 - 2 } Odds ratio $(95 \%$ CI $)$ & $P$ value \\
\cline { 1 - 2 } $0.987(0.973-1.001)$ & 0.063 \\
$1.347(0.864-2.100)$ & 0.189 \\
$0.971(0.947-0.997)$ & 0.029 \\
& \\
1.000 (reference) & \\
$0.682(0.391-1.189)$ & 0.177 \\
$0.726(0.399-1.319)$ & 0.293 \\
$0.931(0.476-1.821)$ & 0.835 \\
& \\
$1.446(0.987-2.117)$ & 0.058 \\
$1.451(1.008-2.087)$ & 0.045 \\
$0.121(0.063-0.232)$ & $<0.001$ \\
& \\
$2.146(0.875-5.263)$ & 0.095 \\
$0.709(0.395-1.272)$ & 0.248 \\
$1.512(0.846-2.702)$ & 0.163 \\
$0.977(0.898-1.062)$ & 0.581 \\
$0.985(0.941-1.031)$ & 0.510 \\
&
\end{tabular}

Dose escalation of GC Odds ratio $(95 \% \mathrm{CI}) \quad P$ value $\underline{\text { Baseline factor }}$

Age, years

Body weight, $\mathrm{kg}$

Steinbrocker's stage I

II

III

IV

Steinbrocker's class

I

II

IV

Previous treatment Bio-experienced Bio-failure

GC GC
csDMARDs (except MTX) $\triangle$ Dose escalation of - Dose reduction of GC

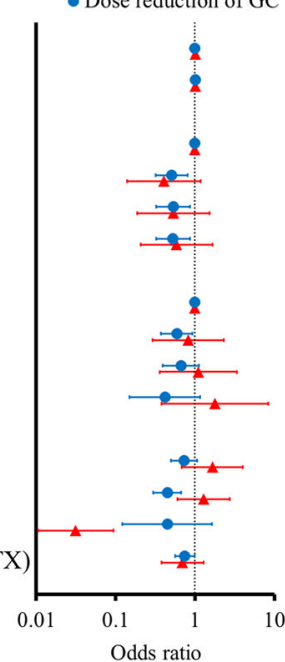

$1.003(0.980-1.027) \quad 0.769$

$\begin{array}{ll}1.016(0.991-1.042) & 0.202\end{array}$

1.000 (reference)

$0.406(0.140-1.174) \quad 0.096$

$0.532(0.186-1.525) \quad 0.240$

$0.584(0.206-1.653) \quad 0.311$

1.000 (reference)

$0.821(0.293-2.300) \quad 0.707$

$1.110(0.362-3.400) \quad 0.855$

$1.774(0.377-8.345) \quad 0.468$

$1.663(0.683-4.045) \quad 0.262$

$1.283(0.598-2.750) \quad 0.522$

$0.031(0.011-0.094)<0.001$

$0.694(0.376-1.281) \quad 0.243$
Dose reduction of MTX

Odds ratio $(95 \% \mathrm{CI}) \quad P$ value

$0.999(0.984-1.014) \quad 0.899$

$0.665(0.389-1.136) \quad 0.135$

$0.980(0.955-1.006) \quad 0.123$

1.000 (reference)

$0.756(0.436-1.311) \quad 0.319$

$0.588(0.316-1.093) \quad 0.093$

$1.103(0.570-2.135) \quad 0.771$

$0.517(0.343-0.779) \quad 0.002$

$0.848(0.584-1.231) \quad 0.386$

$2.443(0.322-18.541) \quad 0.388$

$3.312(1.461-7.508) \quad 0.004$

$1.554(0.954-2.530) \quad 0.077$

$1.146(0.620-2.119) \quad 0.663$

$0.936(0.848-1.034) \quad 0.193$

$0.984(0.937-1.034) \quad 0.521$
Dose reduction of $\mathrm{GC}$

Odds ratio $(95 \% \mathrm{CI}) \quad P$ value

$0.990(0.979-1.002) \quad 0.090$

$1.012(0.999-1.025) \quad 0.063$

1.000 (reference)

$0.510(0.320-0.813) \quad 0.005$

$0.532(0.327-0.867) \quad 0.011$

$0.529(0.323-0.869) \quad 0.012$

1.000 (reference)

$0.588(0.371-0.931) \quad 0.023$

$0.666(0.393-1.129) \quad 0.131$

$0.417(0.149-1.169) \quad 0.096$

$0.731(0.499-1.070) \quad 0.107$

$0.446(0.296-0.674)<0.001$

$0.447(0.122-1.628) \quad 0.222$

$0.747(0.558-0.999) \quad 0.049$ 
4Fig. 3 Forest plots illustrating patient background factors associated with changing the dosage of golimumab (a), methotrexate (b), and glucocorticoids (c). Univariate logistic regression analysis was performed using demographic factors that showed $p<0.10$ in other analyses, after which multivariate analysis was conducted by using variables showing $p<0.20$ in the univariate analysis. To identify the factors associated with changes of MTX and GC therapy (continuing the same dose vs. dose escalation or dose reduction), a multinomial logistic model [35] was used. Error bars indicate the $95 \% \mathrm{CI}$

optimization were recommended [29]. Moreover, renal impairment was associated with MTX dose reduction. Among patients with renal impairment, the subgroup with MTX dose reduction had a higher frequency of overall adverse events $(66.7 \%, 8 / 12)$ than the subgroup with continue same dose of MTX $(0.0 \%, 0 / 30)$, suggesting that adverse events also led to reducing the dosage of MTX. In any event, these results indicate that many physicians adjusted the MTX dosage to control RA symptoms and/ or to prevent adverse events. However, the average dose of MTX was not decreased during the study period of 24 weeks. This might have been because MTX is recognized as a 'first-line treatment' and 'anchor drug' for RA worldwide and there is abundant evidence about its efficacy available [30]. However, adverse effects were reported in $27 \%$ of RA patients receiving low-dose MTX (10 mg/week for at least 3 months), especially hepatic and hematological toxicities [31], and several studies have suggested that tapering or discontinuing MTX does not reduce the efficacy of combination therapy for RA [32, 33]. Because patients and physicians often wish to discontinue MTX due to various adverse events, further investigation will be required to clarify the optimum GLM +MTX regimen for RA.

GC are also often used in combination therapy with bDMARDs. In this study, only previous GC use was associated with selection of concomitant GC therapy at the initiation of GLM treatment. As with MTX, almost all previous GC users continued the concomitant administration of GC with GLM (96.6\%, 1203/1245). Interestingly, the average GC dose was significantly reduced during the study period, suggesting that GC can be tapered in patients receiving GLM. The factors associated with GC dose reduction were a Steinbrocker's stage I, absence of bio-failure, and no previous csDMARDs (except MTX) use. The clinical response of the patients with GC dose reduction was better than that of the other subgroups, suggesting that physicians might have tapered or discontinued GC therapy in patients with stable RA, especially in the patients with Steinbrocker's stage I, no bio-failure, and/or no previous csDMARDs use at baseline. However, the majority of patients did not discontinue GC (a)

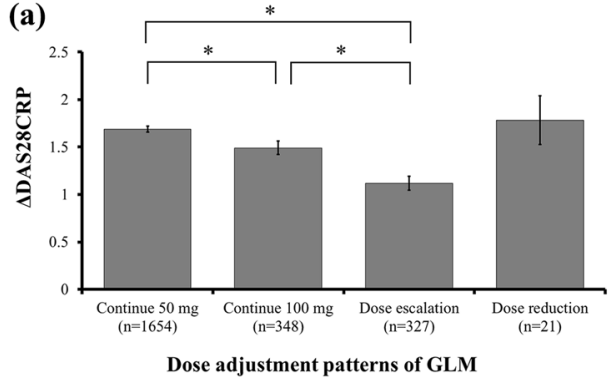

(b)

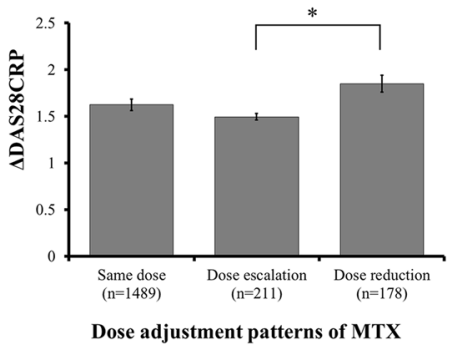

(c)

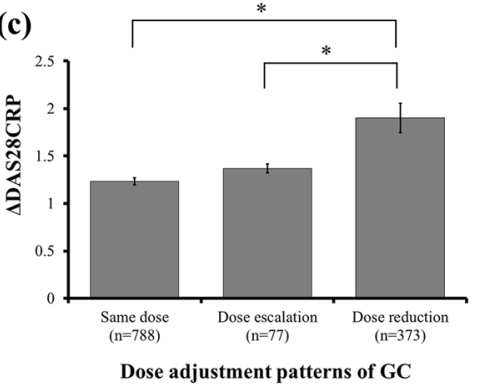

Fig. 4 Clinical response in each of the golimumab (a), methotrexate (b), and glucocorticoids (c) treatment subgroups. Differences of the DAS28CRP score between baseline and final evaluation $(\triangle \mathrm{DAS} 28 \mathrm{CRP})$ are shown in relation to golimumab, methotrexate, and/or glucocorticoids administration. Statistical significance was assessed between the subgroups by ANOVA or the Kruskal-Wallis test for continuous variables, while the Tukey-Kramer method was used to compare multiple continuous variables within subgroups. Final evaluation was defined as the last 4 weeks of the study period. Error bars indicate the standard error (SE). ${ }^{*} p<0.05$ 
$(64.6 \%, 788 / 1219)$ during the study period of 24 weeks. It was reported that some patients who start GC therapy are unable to discontinue it over the long term, despite addition of a csDMARD early in the course to decrease the risk of flare-up and obtain a disease-modifying effect [34]. Further evidence is needed with regard to symptomatic improvement without flare-ups or adverse events when bDMARD therapy is added to reduce the GC dose.

The present study documented current treatment patterns for administrations of GLM in Japan, but several important limitations should be noted. First, although this study was based on PMS data obtained over 24 weeks in the real-world clinical setting, some patients were excluded according to the criteria in the Methods section or for other reasons (e.g., patients who stopped GLM treatment due to safety problems). Second, treatment selection should be affected by local labeling. For example, monotherapy with $50 \mathrm{mg}$ of GLM is off-label in Japan, which may have led to selection of the 100-mg dose or concomitant use of MTX to reduce costs rather than on the basis of the clinical response. Therefore, the generalizability of our result on the treatment selection should have a limitation and further evidence, especially from other or multi countries will be essential for revealing the effect of dose difference. Finally, most analyses were performed within subgroups based on the original PMS data, so interpretation and generalization of associations among the factors assessed and the treatment patterns should be performed with caution. No causal relationship with outcomes or prediction of the effects of a particular treatment pattern can be derived from our research, so further studies are needed to clarify such relationships.

\section{CONCLUSIONS}

In conclusion, this study revealed that various baseline patient factors were associated with initiation of GLM treatment and dose adjustment in RA patients receiving GLM with/without concomitant medications. It is thought that these factors may reflect both the treatment strategies of physicians for improving RA symptoms and/or reducing adverse events, as well as the wishes of patients. Add-on therapy with GLM had the potential to achieve GC and MTX dose reduction with improvement of RA symptoms. However, completely discontinuing concomitant medications, especially MTX, seems to be difficult, suggesting that further evidence is required to determine the safest and most effective strategy for managing RA with GLM.

\section{ACKNOWLEDGEMENTS}

Funding. Sponsorship for this study and article processing charges were jointly provided by Janssen Pharmaceutical K.K. (Tokyo, Japan) and Mitsubishi Tanabe Pharma Corporation (Osaka, Japan). All authors had full access to all of the data in this study and take complete responsibility for the integrity of the data and accuracy of the data analysis.

Authorship. All named authors meet the International Committee of Medical Journal Editors (ICMJE) criteria for authorship for this article, take responsibility for the integrity of the work as a whole, and have given their approval for this version to be published.

Medical Writing and/or Editorial Assistance. The authors would like to deeply acknowledge all patients and physicians who participated in the PMS study for dedication. The authors also wish to thank Toyokazu Saiki, Toshiro Yano, Kenichirou Watanabe, Takahiro Esaki, Kazuhiro Fukuda, and Takashi Inui (Ikuyaku. Integrated Value Development Division, Mitsubishi Tanabe Pharma Corporation) for their contribution to study design and data interpretation.

Disclosures. Masateru Okazaki is an employee of Janssen Pharmaceutical K.K. and may hold stock and/or stock options in the company. Hisanori Kobayashi is an employee of Janssen Pharmaceutical K.K. and may hold stock and/or stock options in the company. 
Yutaka Ishii is an employee of Janssen Pharmaceutical K.K. and may hold stock and/or stock options in the company. Masayoshi Kanbori is an employee of Janssen Pharmaceutical K.K. and may hold stock and/or stock options in the company. Tsutomu Yajima is an employee of Janssen Pharmaceutical K.K. and may hold stock and/or stock options in the company.

Compliance with Ethics Guidelines. This article is based on previously conducted PMS study and does not contain any new interventional studies with human participants or animal subjects performed by any of the authors. The PMS study protocol and ethical considerations were assessed by the internal review board members and approved by the Japanese PMDA. The PMS was conducted under the Japanese regulations (Ministry of Health, Labor and Welfare Ministerial Ordinance No. 171) for Good Post-Marketing Study Practice (GPSP), and was initiated after a contract with each study center.

Data Availability. The datasets generated during and/or analyzed during the current study are not publicly available due confidentiality clauses signed with participating medical institutions.

Open Access. This article is distributed under the terms of the Creative Commons Attribution-NonCommercial 4.0 International License (http://creativecommons.org/licenses/ by-nc/4.0/), which permits any noncommercial use, distribution, and reproduction in any medium, provided you give appropriate credit to the original author(s) and the source, provide a link to the Creative Commons license, and indicate if changes were made.

\section{REFERENCES}

1. Yamanaka H, Sugiyama N, Inoue E, Taniguchi A, Momohara S. Estimates of the prevalence of and current treatment practices for rheumatoid arthritis in Japan using reimbursement data from health insurance societies and the IORRA cohort (I). Mod Rheumatol. 2014;24(1):33-40.
2. McInnes IB, Schett G. The pathogenesis of rheumatoid arthritis. N Engl J Med. 2011;365(23):2205-19.

3. Aletaha D, Neogi T, Silman AJ, Funovits J, Felson DT, Bingham CO 3rd, et al. 2010 Rheumatoid arthritis classification criteria: an American College of Rheumatology/European League Against Rheumatism collaborative initiative. Arthritis Rheum. 2010;62(9):2569-81.

4. Rossini M, De Vita S, Ferri C, Govoni M, Paolazzi G, Salvarani C, et al. Golimumab: a novel anti-tumor necrosis factor. Biol Ther. 2013;3(2):83-107.

5. Zidi I, Bouaziz A, Mnif W, Bartegi A, Al-Hizab FA, Amor NB. Golimumab therapy of rheumatoid arthritis: an overview. Scand J Immunol. 2010;72(2):75-85.

6. Keystone EC, Genovese MC, Klareskog L, Hsia EC, Hall ST, Miranda PC, et al. Golimumab, a human antibody to tumour necrosis factor alpha given by monthly subcutaneous injections, in active rheumatoid arthritis despite methotrexate therapy: the GO-FORWARD Study. Ann Rheum Dis. 2009;68(6):789-96.

7. Kavanaugh A, McInnes I, Mease P, Krueger GG, Gladman D, Gomez-Reino J, et al. Golimumab, a new human tumor necrosis factor alpha antibody, administered every four weeks as a subcutaneous injection in psoriatic arthritis: twenty-four-week efficacy and safety results of a randomized, placebocontrolled study. Arthritis Rheum. 2009;60(4):976-86.

8. Inman RD, Davis JC Jr, Heijde D, Diekman L, Sieper J, Kim SI, et al. Efficacy and safety of golimumab in patients with ankylosing spondylitis: results of a randomized, double-blind, placebo-controlled, phase III trial. Arthritis Rheum. 2008;58(11):3402-12.

9. Sieper J, van der Heijde D, Dougados M, Maksymowych WP, Scott BB, Boice JA, et al. A randomized, double-blind, placebo-controlled, sixteenweek study of subcutaneous golimumab in patients with active nonradiographic axial spondyloarthritis. Arthritis Rheumatol. 2015;67(10):2702-12.

10. Tanaka $Y$, Harigai $M$, Takeuchi T, Yamanaka $H$, Ishiguro $\mathrm{N}$, Yamamoto $\mathrm{K}$, et al. Golimumab in combination with methotrexate in Japanese patients with active rheumatoid arthritis: results of the GO-FORTH study. Ann Rheum Dis. 2012;71(6):817-24.

11. Takeuchi T, Harigai M, Tanaka Y, Yamanaka $H$, Ishiguro N, Yamamoto $\mathrm{K}$, et al. Golimumab monotherapy in Japanese patients with active rheumatoid arthritis despite prior treatment with 
disease-modifying antirheumatic drugs: results of the phase $2 / 3$, multicentre, randomised, doubleblind, placebo-controlled GO-MONO study through 24 weeks. Ann Rheum Dis. 2013;72(9):1488-95.

12. Kanbori M, Suzuka H, Yajima T, Kishino E, Morishige $\mathrm{R}$, Momohara $\mathrm{S}$, et al. Postmarketing surveillance evaluating the safety and effectiveness of golimumab in Japanese patients with rheumatoid arthritis. Mod Rheumatol. 2018;28(1):66-75.

13. Smolen JS, Landewe R, Bijlsma J, Burmester G, Chatzidionysiou $\mathrm{K}$, Dougados $\mathrm{M}$, et al. EULAR recommendations for the management of rheumatoid arthritis with synthetic and biological diseasemodifying antirheumatic drugs: 2016 update. Ann Rheum Dis. 2017;76(6):960-77.

14. Smolen JS, Kay J, Doyle MK, Landewe R, Matteson EL, Wollenhaupt J, et al. Golimumab in patients with active rheumatoid arthritis after treatment with tumour necrosis factor alpha inhibitors (GOAFTER study): a multicentre, randomised, doubleblind, placebo-controlled, phase III trial. Lancet. 2009;374(9685):210-21.

15. van der Heijde D, Klareskog L, Rodriguez-Valverde $\mathrm{V}$, Codreanu C, Bolosiu H, Melo-Gomes J, et al. Comparison of etanercept and methotrexate, alone and combined, in the treatment of rheumatoid arthritis: two-year clinical and radiographic results from the TEMPO study, a double-blind, randomized trial. Arthritis Rheum. 2006;54(4):1063-74.

16. Breedveld FC, Weisman MH, Kavanaugh AF, Cohen SB, Pavelka K, van Vollenhoven R, et al. The PREMIER study: a multicenter, randomized, doubleblind clinical trial of combination therapy with adalimumab plus methotrexate versus methotrexate alone or adalimumab alone in patients with early, aggressive rheumatoid arthritis who had not had previous methotrexate treatment. Arthritis Rheum. 2006;54(1):26-37.

17. Klareskog L, van der Heijde D, de Jager JP, Gough A, Kalden J, Malaise M, et al. Therapeutic effect of the combination of etanercept and methotrexate compared with each treatment alone in patients with rheumatoid arthritis: double-blind randomised controlled trial. Lancet. 2004;363(9410):675-81.

18. Furst DE, Kremer JM. Methotrexate in rheumatoid arthritis. Arthritis Rheum. 1988;31(3):305-14.

19. Prevoo MLL, Van'T Hof MA, Kuper HH, Van Leeuwen MA, Van De Putte LBA, Van Riel PLCM. Modified disease activity scores that include twentyeight-joint counts development and validation in a prospective longitudinal study of patients with rheumatoid arthritis. Arthritis Rheum. 1995;38(1):44-8.
20. Steinbrocker O, Traeger $\mathrm{CH}$, Batterman RC. Therapeutic criteria in rheumatoid arthritis. J Am Med Assoc. 1949;140(8):659-62.

21. A language and environment for statistical computing $\mathrm{R}$ Foundation for Statistical Computing, Vienna: R Core Team; 2016. https://www.R-project. org/.

22. Zhou H, Jang H, Fleischmann RM, Bouman-Thio E, $\mathrm{Xu} \mathrm{Z}$, Marini JC, et al. Pharmacokinetics and safety of golimumab, a fully human anti-TNF-alpha monoclonal antibody, in subjects with rheumatoid arthritis. J Clin Pharmacol. 2007;47(3):383-96.

23. $\mathrm{Xu} \mathrm{Z,} \mathrm{Vu} \mathrm{T,} \mathrm{Lee} \mathrm{H}, \mathrm{Hu} \mathrm{C}$, Ling J, Yan $\mathrm{H}$, et al. Population pharmacokinetics of golimumab, an anti-tumor necrosis factor-alpha human monoclonal antibody, in patients with psoriatic arthritis. J Clin Pharmacol. 2009;49(9):1056-70.

24. Becciolini A, Biggioggero M, Favalli EG. The role of methotrexate as combination therapy with etanercept in rheumatoid arthritis: retrospective analysis of a local registry. J Int Med Res. 2016;44(1 suppl):113-8.

25. Kashiwazaki S, Ichikawa Y, Sugawara S, Nagaya I, Kawai S, Hakota M, et al. Determination of the clinical optimal dose of L-377 (methotrexate capsule) for the treatment of rheumatoid arthritis. Ensho. 1996;16(6):437-58.

26. Japan College of Rheumatology 2016 guidelines for the use of methotrexate (MTX) in rheumatoid arthritis Japan College of Rheumatology Subcommittee on Development of Guidelines for the Use of Methotrexate in the Treatment of Rheumatoid Arthritis: Yodosha; 2016. http://www.ryumachi-jp. com/publication/pdf/MTX2016kanni.pdf.

27. Frei E 3rd, Blum RH, Pitman SW, Kirkwood JM, Henderson IC, Skarin AT, et al. High dose methotrexate with leucovorin rescue. Rationale and spectrum of antitumor activity. Am J Med. 1980;68(3):370-6.

28. Alarcon GS, Kremer JM, Macaluso M, Weinblatt ME, Cannon GW, Palmer WR, et al. Risk factors for methotrexate-induced lung injury in patients with rheumatoid arthritis. A multicenter, case-control study. Methotrexate-Lung Study Group. Ann Intern Med. 1997;127(5):356-64.

29. Combe B, Landewe R, Daien CI, Hua C, Aletaha D, Alvaro-Gracia JM, et al. 2016 update of the EULAR recommendations for the management of early arthritis. Ann Rheum Dis. 2017;76(6):948-59.

30. Braun J. Methotrexate: optimizing the efficacy in rheumatoid arthritis. Ther Adv Musculoskelet Dis. 2011;3(3):151-8. 
31. Attar SM. Adverse effects of low dose methotrexate in rheumatoid arthritis patients. A hospital-based study. Saudi Med J. 2010;31(8):909-15.

32. Manders SH, van de Laar MA, Rongen-van Dartel SA, Bos R, Visser H, Brus HL, et al. Tapering and discontinuation of methotrexate in patients with RA treated with TNF inhibitors: data from the DREAM registry. RMD Open. 2015;1(1):e000147.

33. Emery P, Hammoudeh M, FitzGerald O, Combe B, Martin-Mola E, Buch MH, et al. Sustained remission with etanercept tapering in early rheumatoid arthritis. N Engl J Med. 2014;371(19):1781-92.

34. Makol A, Davis JM, Crowson CS, Therneau TM, Gabriel SE, Matteson EL. Time trends in glucocorticoid use in rheumatoid arthritis: results from a population-based inception cohort, 1980-1994 versus 1995-2007. Arthritis Care Res. 2014;66(10):1482-8.

35. Venables WN, Ripley BD. Modern applied statistics with S. In: Poisson and Multinomial Models. New York: Springer; 2002. pp. 199-205. 